\title{
Aspect-Oriented Analysis for Software Product Lines Requirements Engineering
}

\author{
Patrícia Varela, João Araújo, \\ $\mathrm{CITI} / \mathrm{FCT}$, \\ Universidade Nova de Lisboa, \\ 2829-516 Caparica, Portugal \\ patixinha@gmail.com, ja@di.fct.unl.pt
}

\author{
Isabel Brito, \\ Instituto Politécnico de Beja, \\ 7800-050, Beja, Portugal \\ issb@estig.ipbeja.pt
}

\author{
Ana Moreira, \\ CITI/FCT, \\ Universidade Nova de Lisboa, \\ 2829-516 Caparica, Portugal \\ amm@di.fct.unl.pt
}

\begin{abstract}
Requirements analysis and modeling for Software Product Lines demands the use of feature models, but also requires additional models to help identifying, describing, and specifying features. Traditional approaches usually perform this manually and, in general, the identification and modularization of crosscutting features is ignored, or not handled systematically. This hinders requirements change. We propose an aspect-oriented approach for SPL enriched to automatically derive feature models where crosscutting features are identified and modularized using aspectoriented concepts and techniques. This is achieved by adapting and extending the AORA (Aspect-Oriented Requirements Analysis) approach. AORA provides templates to specify and organize requirements based on concerns and responsibilities. A set of heuristics is defined to help identifying features and their dependencies in a product line. A tool was developed to automatically generate the feature model from AORA templates.
\end{abstract}

\section{Categories and Subject Descriptors}

D.2.1 [Software Engineering]: Requirements/Specifications -

languages, methodologies.

\section{General Terms}

Design.

\section{Keywords}

Aspect-Oriented Requirements Analysis, Software Product Lines.

\section{INTRODUCTION}

Requirements Engineering includes the identification, analysis, documentation, validation and management of requirements [15]. A requirement describes functionalities, constraints or quality attributes in software systems. Our focus is on creating a synergy between Software Product Lines Engineering (SPLE) and Requirements Engineering benefiting from their concepts, techniques and tools. Whilst Requirements Engineering

Permission to make digital or hard copies of all or part of this work for personal or classroom use is granted without fee provided that copies are not made or distributed for profit or commercial advantage and that copies bear this notice and the full citation on the first page. To copy otherwise, or republish, to post on servers or to redistribute to lists, requires prior specific permission and/or a fee.

$S A C^{\prime} 11$, March 21-25, 2011, TaiChung, Taiwan.

Copyright 2011 ACM 978-1-4503-0113-8/11/03 ..\$10.00. techniques can be used to elicit and specify domain and application requirements, SPLE captures commonalities and variabilities of product families promoting reuse [16]. Thus, in a medium term, productivity can be increased.

One of the most used Software Product Lines (SPL) techniques to specify requirements and handle their commonalities and variations is feature modeling [8][14]. A limitation of feature models is that they do not provide enough information on each feature, such as behavior and a rationale for dependencies, needing other models to supply that information. Another difficulty is dealing with the crosscutting nature of (parts of) some features. Aspect-oriented (AO) techniques [2] have been used successfully to model crosscutting concerns. A concern refers to any matter of interest of one or more stakeholders [11], and a crosscutting concern is any concern that cuts across other concerns. In the context of this paper a feature is a concern and, therefore, we also talk about crosscutting features. A third limitation is the lack of tools to automatically derive feature models from requirements specifications, be them aspect-oriented or not.

The application of requirements engineering techniques, such as use cases [12], viewpoints [9] and goals [6], has improved SPLE specifications [3][7][10] [13][18][20]. This resulted in documents that provide models expressing different perspectives of the requirements [16], therefore complementing and completing the view of requirements specifications. However, requirements elicitation and analysis for SPL could be enhanced if the modularization of crosscutting features were addressed using aspect-oriented techniques [2].

Aspect-Oriented Requirements Engineering (AORE) ${ }^{1}$ appeared to address this problem by identifying, representing, specifying and composing crosscutting concerns. Crosscutting concerns are encapsulated in separate modules, known as aspects [2][17]. One of the pioneering AORE approaches is AORA (Aspect-Oriented Requirements Analysis) [5]. AORA offers some advantages with respect to other existing approaches: a detailed template specification for all types of concerns (functional, non-functional or crosscutting); a set of concepts and techniques rigorously defined in a metamodel; a set of composition operators to study the impact of a set of concerns over a base; an efficient and

1 The interested reader can refer to http://www.aosdeurope.net/deliverables/d11.pdf for a survey on AORE approaches, or to the Early Aspects portal (www.earlyaspects.net). 
rigorous conflict resolution method; and a supporting tool developed based on the defined metamodel.

We adapt and extend AORA to support SPL development at domain and application engineering levels. The result is the PLAORA approach (Product Lines for Aspect-Oriented Requirements Analysis). PLAORA provides a sound set of heuristics to derive feature models taking into consideration the identification of crosscutting concerns at domain and application engineering levels, offers a tool to systematically and automatically identify and generate common and variable features, and uses the Analytic Hierarchy Process (AHP) [19], a multi-criteria method, to identify and resolve conflicts.

In summary, the aim of this paper is to enrich the development of SPL with the capabilities and advantages of AORA, where the specification of concerns facilitates the automatic derivation of a feature model.

This paper is structured as follows. Section 2 summarizes AORA main concepts. Section 3 describes PLAORA. Section 4 applies PLAORA to a case study. Section 5 discusses the evaluation of the approach. Evaluation has been performed using case studies, including an industrial case study, comparing our approach with others, and collecting data from an experiment performed with a group of ten master's students. Section 6 presents some related work, comparing PLAORA with other existing approaches. Finally, Section 7 presents conclusions and future work

\section{BACKGROUND}

\subsection{SPL}

An SPL is a set of software systems, which share common characteristics, satisfying the needs of a particular segment of the market and are developed from a common set of core assets [16]. Domain Engineering and Application Engineering are the two main processes of SPLE. These are complementary activities, interacting as parallel processes forming a base model of a software system. Domain Engineering identifies the SPL commonalities and variabilities. Application Engineering develops members of the product line through configuration, reusing domain core assets and selecting different sets of variations for each SPL product. As mentioned before, the characteristics of an SPL are often specified using a feature model. A feature model consists of a diagram composed of features and additional information, such as semantic descriptions of each feature variable points, reasons for each feature, priorities and dependence rules [3]. A feature is a property of a system relevant for some stakeholders and is used to capture common characteristics or variables in SPL. The types of features, defined are mandatory, optional and alternative.

\subsection{AORA}

AORA [5] is composed of three main tasks: identify concerns, specify concerns and compose concerns. These tasks are accomplished iteratively and incrementally. Identify concerns aims at discovering the concerns of a system, where a concern is as a set of coherent requirements defining a property that the future system must provide. This is performed by analyzing the initial requirements, transcripts of stakeholders' interviews, etc. Good sources for concern identification are the existing catalogues, such as the NFR catalogue [6]. Specify concerns provides textual and visual representations of concerns and their relationships. All the useful information about a concern is collected in a template (Tables 1 and 2 are examples). Finally, Compose concerns offers the possibility to compose a set of concerns, incrementally, until the whole system is obtained (if we need that) and, at the same time, identify the impact of a set of concerns on a given base. A composition occurs in a match point which lists the crosscutting concerns that should be composed with each (set of) base concern, forming a composition rule. A composition rule is formed of concerns and pre-defined operators.

\section{THE PLAORA APPROACH}

Being in tune with SPLE, PLAORA also distinguishes between Domain Engineering and Application Engineering. The product line is created at the Domain Engineering level (according to the process depicted in the top part of Fig.1), and then a product is configured at the Application Engineering level (bottom of Fig.1).

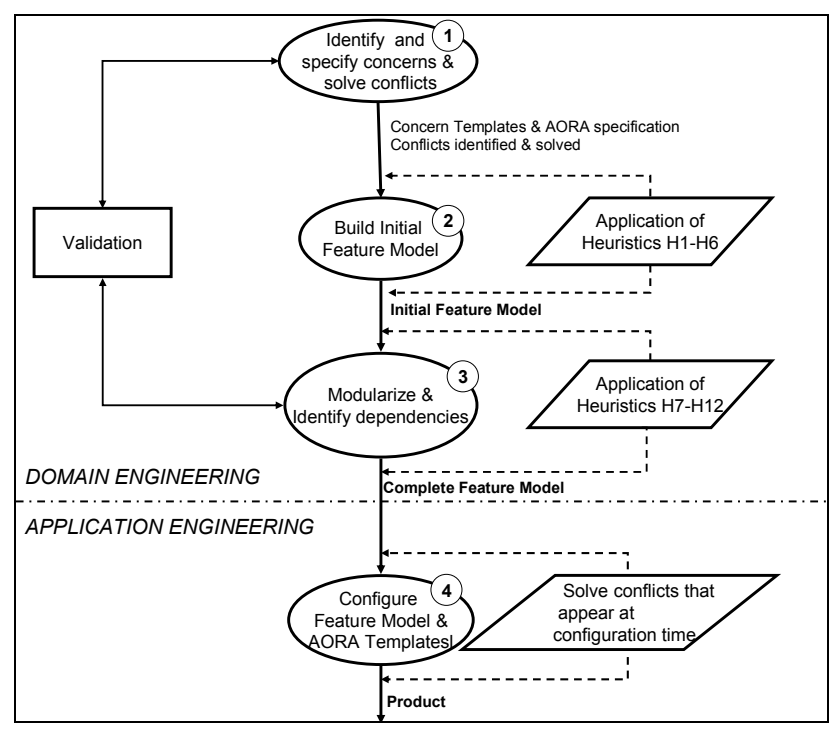

Figure 1 PLAORA process.

The Domain Engineering part of the process initially consists of the identification and specification of system concerns and the conflict identification and resolution (step 1 in Fig. 1). The specification of concerns can lead us to identify new concerns and refine others previously identified. Next, the specification templates are described and then the composition of concerns is realized. Here conflict identification and resolution are carried out using a multicriteria method as described in [4][5] where concerns are ranked according to their importance to different stakeholders. We will not focus on this, as this is not the contribution of this paper.

Having all the concerns specified we can identify the features of the SPL whose result is an initial feature model (step 2 in Fig. 1). These are extracted from AORA templates with the help of a set of heuristics. So features are derived from concern templates, with their lists of responsibilities. The resulting feature model is then refined with a complementary set of heuristics to modularize the feature model and identify dependencies between features (step 3 in Fig. 1). That is, once the extraction of possible features is completed, we identify the variability of the SPL and the different kinds of dependencies between features taking into account 
crosscutting concerns. The heuristics are described and exemplified in Section 4.

Validation must be performed in parallel with the process just described. In particular, stakeholders need to help validating (i) the identified concerns and respective specifications, as well as (2) guaranteeing that the feature model meets their needs.

At the end of domain engineering process, we have concern templates and a feature model representing common and variable requirements in the SPL. These templates and feature models are analyzed and configured for a particular product of the SPL by the Application Engineering process (step 4). Conflicts particular to a specific configuration should be resolved here. Again we do not discuss this here as it is out of scope of this paper.

\section{APPLYING PLAORA AND DESCRIBING THE DEVELOPMENT HEURISTICS}

PLAORA has been applied to two case studies, the Mobile Phone $^{2}$ and the Smart Home ${ }^{3}$ case studies. The Smart Home case study was developed in the AMPLE project [1] and is not described here due to space constraints.

The Mobile Phone case study is used to illustrate our approach. The example's aim is to develop software components to make and answer calls, put phone calls on hold, insert contacts in a contacts list, send and receive e-mails, SMS and MMS, take pictures, set alarm and transfer data between two mobile phones. Payments can be performed by ATMs or banks' websites.

\subsection{Domain Engineering}

For the activities of Domain Engineering process presented in Fig.1, we will focus on the major contribution of our approach: building the initial and final feature model of an SPL. Due to lack of space, let us assume that the modeling system' concerns was already performed and a list of concern templates provided. The functional concerns for our problem are: Make call, Answer call, Put phone call on hold, Enable voice mail, Receive MMS/SMS/Email and Send MMS/SMS/E-mail. Also the non-functional concerns are: Response Time, Usability, Correction, Confidentiality, Availability, Integrity and Security. Two AORA template examples, one functional and another non-functional, are shown in Table 1 and Table 2, respectively.

Table 1. Template for "Make call"concern.

\begin{tabular}{|l|l|}
\hline Name & Make call \\
\hline Sources & $\begin{array}{l}\text { Knowledge of mobile phone software systems, } \\
\text { set of initial requirements, stakeholders }\end{array}$ \\
\hline Stakeholders & User, Mobile Phone Operator \\
\hline Description & $\begin{array}{l}\text { The user answers a call made to his mobile } \\
\text { phone }\end{array}$ \\
\hline Decomposition & $<$ None $>$ \\
\hline Classification & Functional \\
\hline
\end{tabular}

${ }^{2}$ The complete specification can be found in http://ctp.di.fct.unl.pt/ ja/MobilePhone_CaseStudy.pdf.

${ }^{3}$ The complete specification can be found in http://ctp.di.fct.unl.pt/ ja/SmartHome_CaseStudy.pdf.

\begin{tabular}{|l|}
\hline List of responsibilities \\
\hline 1. The call is redirected by Mobile Phone Operator \\
2. Play signal (at least one of the alternatives, sound or vibration) \\
3. The screen displays the phone number \\
4. Push button (only one of the alternatives: accept or reject) \\
5. The call duration appears on the display \\
6. Choose loud voice mode, if desired \\
7. The call is disconnected, after finishing the conversation \\
\hline List of contributions \\
\hline <None> \\
\hline List of priorities \\
1. User: Important \\
2. Mobile Phone Operator: Very Important \\
\hline List of required concerns \\
\hline 1. Usability
\end{tabular}

Table 2. Template for "Response Time" concern.

\begin{tabular}{|c|c|}
\hline Name & Response Time \\
\hline Sources & $\begin{array}{l}\text { Knowledge of mobile phones software system, } \\
\text { set of initial requirements, stakeholders, } \\
\text { documentation, NFR Framework catalogue }\end{array}$ \\
\hline Stakeholders & $\begin{array}{l}\text { User, Mobile Phone Operator, Banking System, } \\
\text { Sender / Receiver }\end{array}$ \\
\hline Description & $\begin{array}{l}\text { The system must react in time, when users want } \\
\text { to use mobile phone functionalities }\end{array}$ \\
\hline Decomposition & $<$ None $>$ \\
\hline Classification & Non-Functional \\
\hline \multicolumn{2}{|c|}{ List of responsibilities } \\
\hline \multicolumn{2}{|c|}{$\begin{array}{l}\text { 1. The system reacts in time to establish the call } \\
\text { 2. The system reacts in time to check if the time of holding the call } \\
\text { reached the limit } \\
\text { 3. The system reacts in time to capture images } \\
\text { 4. The system reacts in time to alert if SMS/MMS/e-mail were } \\
\text { successfully sent } \\
\text { 5. The system reacts in time to alert that if SMS/MMS/e-mail were } \\
\text { received } \\
\text { 6. The system reacts in time to search for devices within a range of the } \\
\text { phone } \\
\text { 7. The system reacts to enable the voice mail }\end{array}$} \\
\hline \multicolumn{2}{|c|}{ List of contributions } \\
\hline \multicolumn{2}{|c|}{$\begin{array}{l}\text { 1. Availability contributes negatively (-) to Response Time } \\
\text { 2. Correctness contributes negatively (-) to Response Time }\end{array}$} \\
\hline \multicolumn{2}{|c|}{ List of priorities } \\
\hline \multicolumn{2}{|c|}{$\begin{array}{l}\text { 1. User: Very Important } \\
\text { 2. Mobile Phone Operator: Very Important } \\
\text { 3. Banking System: Very Important } \\
\text { 4. Sender/Receiver: Very Important }\end{array}$} \\
\hline \multicolumn{2}{|c|}{ List of required concerns } \\
\hline$<$ None $>$ & \\
\hline
\end{tabular}

Heuristics H1-H6 identify the features of the system from the AORA templates. Initially, we assume that all features are mandatory. Heuristics $\mathrm{H} 7-\mathrm{H} 12$ produce a feature model and identify variability.

H1. Identify the root feature based on "sources" entry: Analyze the "Sources" line to get the root feature of the feature model. For example based on source "Knowledge of the mobile phones system", we get the feature "Mobile Phones". Basically, the root name will be the name of the system. 
H2. Identify features based on the concerns' name: Analyze the "Name" line and obtain the system features through these names, i.e., each concern originates a feature. For example, "Make call", "Answer call", "Response Time", "Security" originate features with the same name. To improve readability of the model we proposed a change in the notation on the feature model: features resulting from non-functional concerns are represented by a rectangle with rounded corners, while those from functional concerns are represented by rectangles.

H3. Identify features that can be grouped based on concerns' names: Analyze the "Name" line and make two types of feature groups. (1) Concerns beginning with the same verb, but different objects define a group where the parent feature is composed of the common verb in features plus a generic noun (Verb + Noun). This noun should be generic in order to specify the information common to the sub-feature that you get. For example, considering features "Send SMS", "Send E-mail", "Send MMS", we obtain a parent feature named "Send data", as data is a more generic noun that can be specialized as SMS, MMS or E-mail. As sub-features we have the original "Send SMS", "Send Email" and "Send MMS" features. (2) The same object refers to different verbs. In this case we define a generic verb and use the object that occurs repeatedly originating the parent feature. For example, features "Make call", "Answer Call" and "Put phone call on hold" share the word "call", originating a group where we can define "Processing call" as a parent feature.

H4. Identify features based on concerns' "decomposition": Analyze the "Decomposition" line and, in the case of refinement, the refined concerns are sub-features and the concern that was refined is the parent feature. For example, features "Integrity" and "Confidentiality" are subfeatures of "Security". This refinement is based on the catalogue for security offered in [6].

H5. Identify features based on the "list of responsibilities": Analyze "List of Responsibilities" and choose those starting with a "Verb + Noun" and which play an important role in the system; these may originate new features (or sub-features). For example, "Make call" has sub-features "Dial number", "Push the call button", "Choose a loud voice", taken from responsibilities "Dial number desired", "Push the call button to start call" and "Choose loud voice to the call if desired". Features extracted using this heuristic requires the user intervention to interact with the system. Also, using "List of Responsibilities" check for additional information to be defined as features to represent in the model, like types of information or ways to achieve functionality. For example, "Answer call" has the responsibility "Push button (only one of the alternatives, accept call or reject call)" which gives us the features "accept call", "reject call".

H6. Identify features based on the NFR catalog: Using existing catalogues, such as [9], we can identify new features for the nonfunctional requirements (NFRs). These features will be added to the feature model.

H7. Identify variability from concern's description: The "Description" line identifies an optional feature if a modal verb expresses non-obligatory, such as if "can" or "may" appear in the description. This variability is related to the features extracted from H2. For example, "Put phone call on hold" has the description "The user can place a particular call waiting", which includes "can". Hence, "Put phone call on hold" is optional.

H8. Identify variability for other features of the model: Analyze in "List of Responsibilities" if responsibilities therein have expressions such as "if desired", "if wanted", "if possible", for example; these are optional. The concern "Make call" has the responsibility "Choose loud voice to the call mode, if desired"; therefore, the feature "Choose loud voice" obtained by H5 is classified as optional.

H9. Identify xor alternatives: Analyze "List of Responsibilities" using expressions like "only one of the alternatives"; these are xor alternatives. For example, the concern "Answer call" has a responsibility "Push button (only one of the alternatives, accept call or reject call)". Therefore, the features "Accept call" and "Reject call" are sub-features of the feature "Push button" providing a xor alternative in the model.

H10. Identify or alternatives: Analyze in "List of Responsibilities" expressions such as "at least one of the alternatives"; these are identified as or alternatives. For example, in "Answer call" template, the responsibility "Play signal (at least one of the alternatives, sound or vibration) on your phone", allows the identification of the features "Sound" and "Vibration". These are alternative sub-features of the feature "Play signal" providing or alternative in the feature model.

H11. Identify requires dependencies relationships in feature model: "List of required concerns" in the template originates requires dependencies relationships, represented by dashed arrows. For example, in the "Answer call" template, the required concern "Usability" originates a require dependency relationship in the feature model. One feature that has more than one arrow, pointing to itself, is identified as a crosscutting feature.

H12. Identify excludes dependencies relationships in feature model: Those responsibilities in "List of Responsibilities" that include expressions like "excluding the possibility of X" originate excludes dependency relationship, where $\mathrm{X}$ identifies the other feature present in the link. For example, the responsibility "The service is active, excluding the possibility of putting phone call on hold" in "Enable voice mail" template originates an excludes dependency relationship in the model between the features "Enable voice mail" and "Put phone call on hold".

The 12 heuristics applied to our case study originated the feature model in Fig.2, where variability is identified. For simplification purposes we represented only requires dependencies relationships for the features "Make Call" and "Answer call" as an example of H11. To reduce the complexity of the feature model with respect to the requires dependencies relationships, we added a small rectangle labeled "requires" under the features (Fig.3) that require others. The list of numbers after "requires" corresponds to the numbers of the required features. This numbering is done from left to right on the model, numbering only the features that were extracted from the names of concerns (H2), as these are required by other concerns. H11 can identify crosscutting features, those that are required by at least another feature. Once we have specified and modularized the SPL features following AO principles, the crosscutting features emerge automatically: they are those represented more than once over the rectangles with a label "requires". Fig.3 identifies "Usability", "Response Time" 
and "Availability" as crosscutting features (these have a black triangle placed at the bottom right hand corner of features, as shown in Fig.3). An example of a crosscutting functional feature is "Mobile phone payment" specified in the URL provided ${ }^{2}$. Note that, the rectangle below the features in Figure 3 has the number of the features that are required, e.g., "Send E-mail" (9) requires Usability (11), and Response Time (12).

\subsection{Application Engineering}

AORA and the heuristics were used to capture the domain engineering features. Now we can choose different configurations, each one representing a different product of the family. Both, the feature model and the AORA templates, are configured for a specific product. Firstly, it is configured the feature model and then the AORA templates.

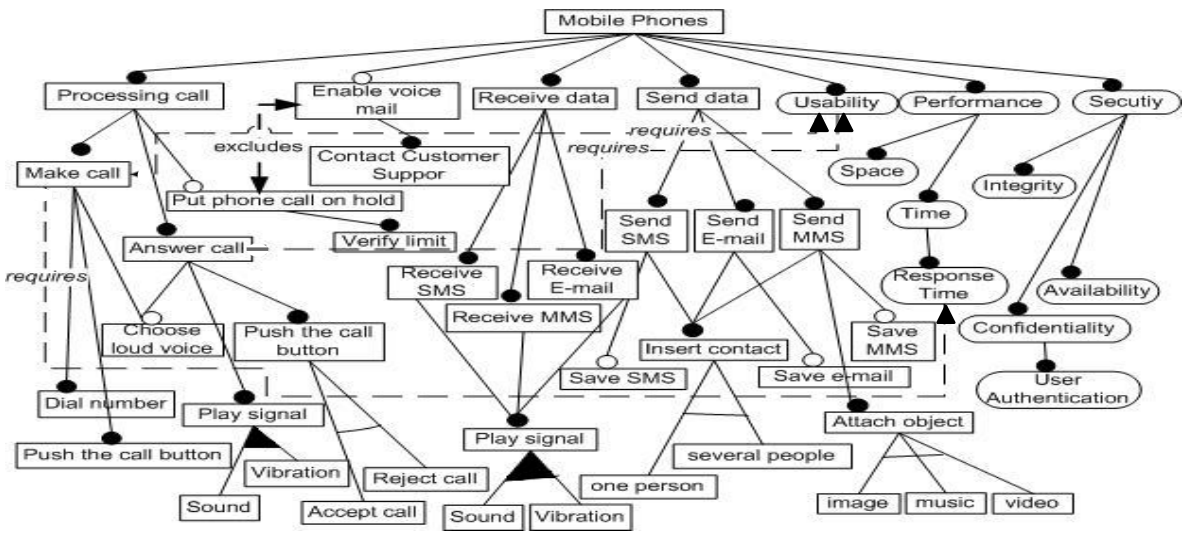

Figure 2. Feature model: a version derived from the application of the $\mathbf{1 2}$ heuristics presented.

As an example we want a mobile phone application with the following functionalities: make/answer calls and send /receive SMS and MMS. Fig. 4 illustrates the feature model of the configured application. Response Time and Usability are crosscutting features, since they are required by several features, recognized with a black triangle placed at the bottom right hand corner of the features.
The changes in the templates are done at the level of responsibilities and description of the concerns since these entries in templates are those used to obtain the system's variability. A concrete application does not include "optional" features, or "alternatives", "or" and "xor". Due to lack of space the configuration of the AORA templates are not presented, but they can be found in the URL provided ${ }^{2}$.

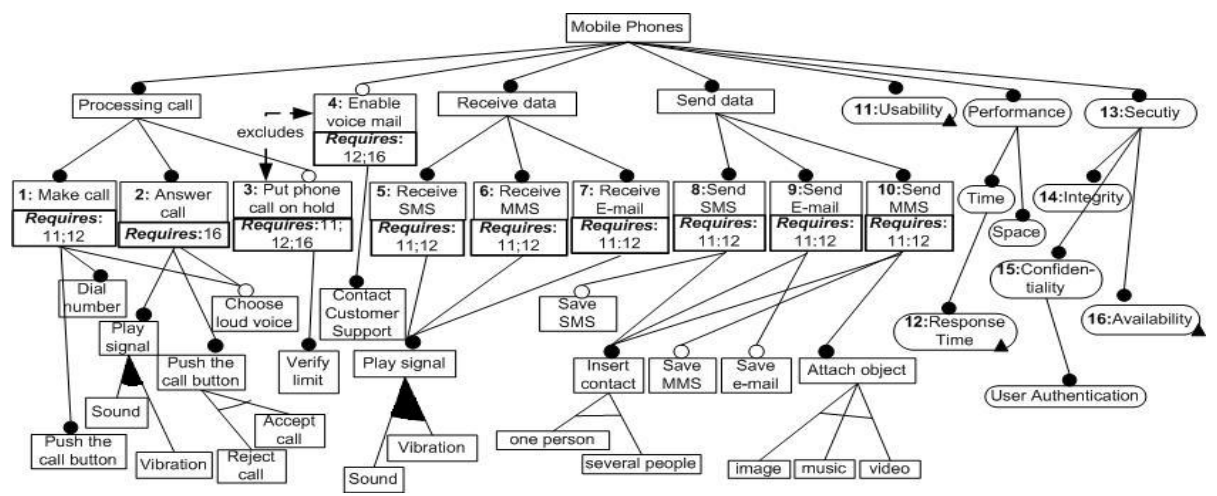

Figure 3. Feature model - final version.

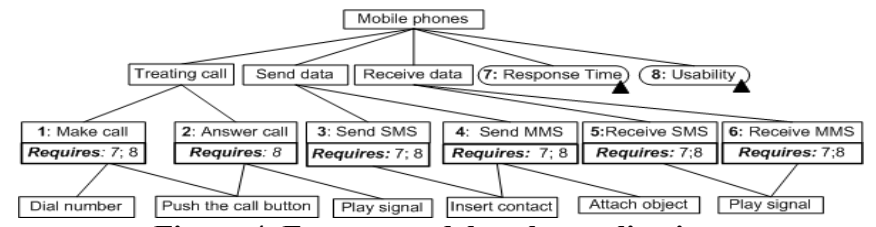

Figure 4. Feature model to the application.

\subsection{Tool support}

The AORA tool specifies and composes concerns, keeping a repository with all the identified elements and relationships. This tool was extended to generate the feature model. This extension implements the 12 heuristics offered by PLAORA. A snapshot of the tool is presented in Fig. 5, showing a feature model. By clicking on the yellow button (marked with circle " 1 " in the red rectangle on the left hand side of figure), the feature model is generated automatically (window on the right).

Tray diamond, marked with circle " 2 " in Fig. 5, represents the root of the model, and the middle of the image shows the mobile phone terms. The features of the system are thus connected by the links "optional", "mandatory", "or", "xor" and "excludes". By selecting the concern and clicking on the button marked with a black triangle, the user can visualize the list of concerns that the selected concern cuts across, as shown in small window in Fig.5 marked with circle " 3 ". The different colors for elements in this diagram indicate the crosscutting features: red elements represent 
crosscutting features and blue elements represent non-crosscutting ones. A parser was implemented for the heuristics to collect information from the repository, which is necessary to extract features of the system. For example, at the responsibilities level, if the first two words that compose the responsibility are a "verb + noun", we obtain the features according to H5. Also, to analyze if the responsibility presents extra information, we need to verify if that information (included between brackets) is useful. The variability analysis is performed by analyzing if the responsibility sentences contain reserved words, such as "if desired/if wanted/if possible", "only one of the alternatives", "at least one of the alternatives" and "excluding the possibility of", which, in that order, originate optional features, xor alternative, or alternative and excludes dependencies relationships (H8-H10, H12) in the feature model. Note that the expressions list is extensible.

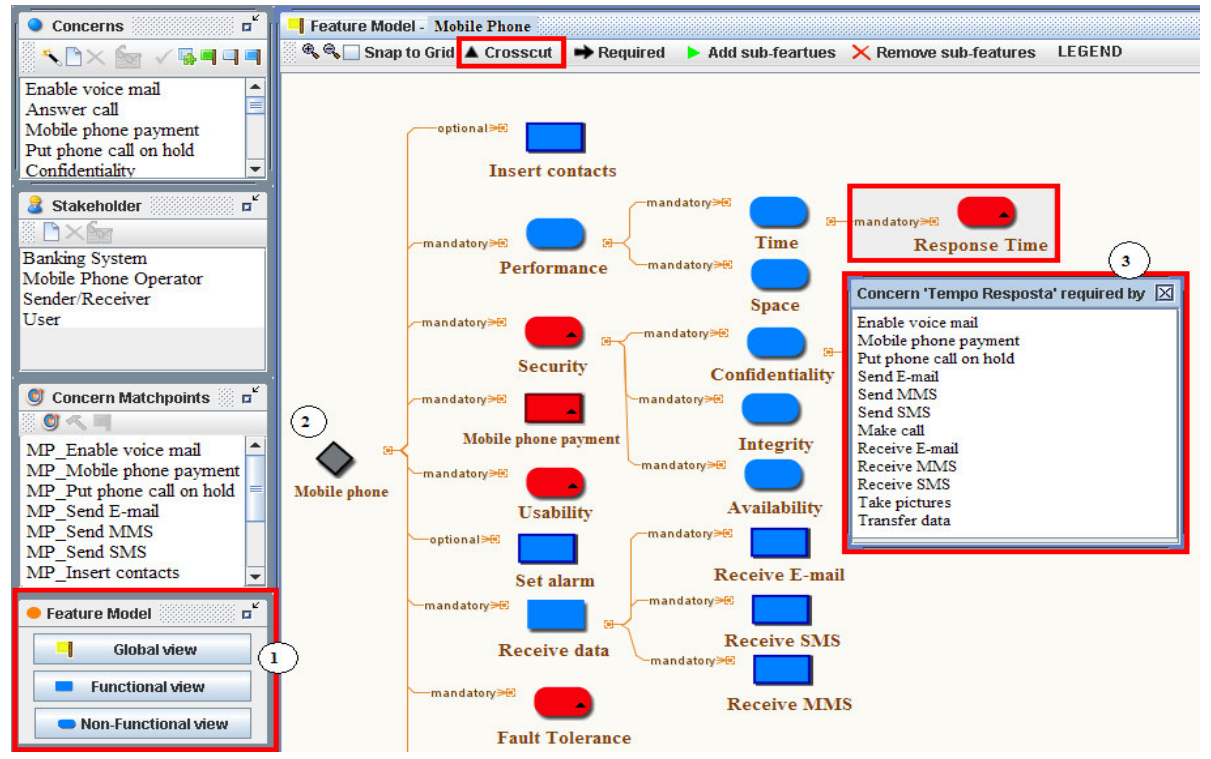

Figure 5. Snapshot of the tool with the feature model.

NFRs are addressed by H6 (for example, "Response Time"). The tool automatically adds the existing information in the corresponding NFR catalogue. For instance, the feature "Performance" is added and, consequently, its sub-features "Time" and "Space". Therefore, "Response Time" is a sub-feature of "Time". Also, if we have the concern "Security", it will always have as sub-features "Integrity", "Confidentiality" and "Availability".

A second parser was implemented to identify optional features (H7) taking into consideration if the description of the concerns included words like "can" or "may". Obviously, the parsers can be extended to accept other expressions that will help to derive the features, their kinds, and their relationships.

\section{EVALUATION}

PLAORA was evaluated in three ways: (1) based both on case studies, in particular, Smart Home ${ }^{3}$ and Health Watcher ${ }^{4 ;}$ (2) based on a questionnaire ${ }^{5}$ answered by 10 MSc students; (3) comparison to other approaches (Section 6.2).

The Smart Home case study helped us find several situations needing improvement. For example, H8 was extended to consider as variability the information provided in the list of responsibilities, which is contained in brackets without reserved

\footnotetext{
${ }^{4}$ The Health Watcher case study can be found in http://ctp.di.fct.unl.pt/ ja/HW_CaseStudy.pdf.

${ }^{5}$ The questionnaire can be found in http://ctp.di.fct.unl.pt/ ja/Questionnaire.pdf.
}

expressions. These reserved expressions are "if desired" (in the original H8), "only one of the alternatives" (H9), and "at least one of the alternatives" (H10), corresponding features will be derived in the model. The new heuristic is illustrated in the "Configure security control system" concern ${ }^{3}$, where we have the responsibility "Simulate presence (define rooms, insert date, set initial time, set end time, set duration, set frequency)" where the features "define room", "insert date", "set initial time", "set end time", "set duration" and "set frequency" are defined as mandatory and also the sub-features of "Simulate presence".

Another issue is the list of numbers after "requires", as shown in Fig. 4. In the case of requirements change, all the numbering must be redone. This problem can be solved by making the tool capable of reflecting the impact of the change in the model.

The Health Watcher was also used to validate the approach, where a PLAORA specification was given to a set of 10 Master's students with knowledge on SPL and AORE. The students were asked to build individually a feature model based on given specification and then to compare their feature model with the one generated by the tool. At the end they were asked to answer a questionnaire whose questions involved the identification of features, the contribution of templates to identify features and to create the feature model, comparison between the model generated and the model drawn by hand, the advantage of the implemented tool views, the advantage of representing aspects, and the advantage of representing the requires dependency relationships modularly.

The results obtained have the following positive points: (i) existence of the functional and non-functional views on the 
feature model; (ii) ability to expand and collapse features, reducing the complexity of the models; (iii) identify crosscutting features and "requires" dependencies relationships modularization. The negative points are: (i) representation of the syntax of the model to be unabbreviated; and (ii) lack of a legend to help the perception of the various features represented in the model.

Some suggestions for improvement were presented: (i) transform the abstract syntax of models into a standard one; (ii) add in the models a different notation for those features that have the ability to add/remove sub-features; (iii) add a descriptive label in the buttons to add and remove sub-features of a given feature previously selected; (iv) provide a legend to facilitate the understanding of the models. These suggestions were later implemented to improve the tool functionality.

\section{RELATED WORK}

\subsection{Related AO SPL approaches}

Silva et al. present an approach [18] to show that $i^{*}$ extended with aspects can support variability for SPL. Heuristics are presented to map the aspectual $i^{*}$ model to the feature model. However, the approach needs to be improved to manage models' scalability. The approach considers that each feature, optional or alternative is mapped into one aspect and this is not always the case. Our proposal tries to covers these limitations and also offers tool support.
Jayaraman et al. present an approach [13] aiming at maintaining the separation of features during the modeling of systems based on UML models. It also detects unwanted structural interactions between the different types of features. Also, the basic features are expressed in terms of class diagrams, sequence diagrams and state diagrams in UML, while variable features are specified in UMLT (UML Transformation), which is a UML representation of transformations of graphs. Our proposal differs from theirs, since we provide a set of heuristics to derive a feature model and they do not specify a separate feature model.

Bonifácio and Borba present an approach [3] whose main objective is to characterize the management of variability, as a crosscutting concern. The specification of concerns variability is done separately. It suggests a framework for modeling the process of composition of variability in scenarios. This framework provides a basis to describe variability as aspects mechanisms, differently from existing approaches, since it considers the contribution of different input languages. It presents the specification of three forms of variability for use case scenarios, such as, variability in function, variability in data and variability in flow control. Our proposal differs from theirs as we offer a set of heuristics to identify features, create feature models and help identifying variability

\subsection{A comparative study}

The aspectual SPL approaches described in Section 5.1 are now compared with PLAORA. Table 3 summarizes the results of the comparison.

Table 3. Comparison between AO approaches integrated in SPL.

\begin{tabular}{|c|c|c|c|c|}
\hline Criteria ${ }^{\text {Approach }}$ & PLAORA & $\begin{array}{c}\text { Aspectual I* \& SPL } \\
{[20]}\end{array}$ & MATA \& SPL [13] & $\begin{array}{c}\text { Use cases and Feature Models } \\
{[3]}\end{array}$ \\
\hline $\begin{array}{l}\text { Conflict } \\
\text { resolution }\end{array}$ & $\begin{array}{l}\text { Offers rigorous decision support system } \\
\text { to identify (using contribution and } \\
\text { priorities) and solve conflicts with AHP } \\
\text { at a more abstract level. }\end{array}$ & $\begin{array}{l}\text { Can be extended to } \\
\text { support the negative } \\
\text { contribution } \\
\text { relationship as in [6]. }\end{array}$ & $\begin{array}{l}\text { Uses pair-analysis for } \\
\text { identifying conflicts in more } \\
\text { detailed analysis models (e.g. } \\
\text { sequence diagrams). }\end{array}$ & No \\
\hline Heuristics & $\begin{array}{l}\text { For domain and application engineering, } \\
\text { as well as for identifying crosscutting } \\
\text { concerns. }\end{array}$ & $\begin{array}{l}\text { Only used to reduce } \\
\text { the model complexity } \\
\& \text { identification of } \\
\text { crosscutting. }\end{array}$ & No & No \\
\hline $\begin{array}{l}\text { Tool } \\
\text { support }\end{array}$ & $\begin{array}{l}\text { Models composition, variabilities with } \\
\text { feature models, product configuration, } \\
\text { configuration knowledge and conflict } \\
\text { detection. It maintains a repository of } \\
\text { elements \& relationships, where all the } \\
\text { information is kept according to AORA } \\
\text { templates. }\end{array}$ & No & $\begin{array}{l}\text { Allows automated } \\
\text { composition of UML models } \\
\text { of features and detection of } \\
\text { some kinds of feature } \\
\text { interactions. }\end{array}$ & $\begin{array}{l}\text { Models composition of } \\
\text { scenario variabilities with } \\
\text { feature models, product } \\
\text { configuration, and } \\
\text { configuration knowledge. }\end{array}$ \\
\hline Modelling reqs. & Aspect and object oriented. & Aspect and goal driven & Aspect and object oriented. & Aspect and UC oriented. \\
\hline Modeling features & Captures commonality \& variability. & $\begin{array}{l}\text { Captures commonality } \\
\& \text { variability. }\end{array}$ & $\begin{array}{l}\text { Captures commonality \& } \\
\text { variability. }\end{array}$ & $\begin{array}{l}\text { Captures commonality \& } \\
\text { variability. }\end{array}$ \\
\hline $\begin{array}{r}\text { Modeling } \\
\text { scenarios }\end{array}$ & $\begin{array}{l}\text { Uses UML sequence diagrams and use } \\
\text { cases (from original AORA). }\end{array}$ & No & $\begin{array}{l}\text { Uses UML sequence } \\
\text { diagrams. }\end{array}$ & Provides use cases. \\
\hline Feature interaction & $\begin{array}{l}\text { Identify requires and excludes } \\
\text { dependencies relationships in feature } \\
\text { model }\end{array}$ & No & $\begin{array}{l}\text { Feature interactions can be } \\
\text { verified for consistency with } \\
\text { the relations captured in the } \\
\text { feature dependency diagram }\end{array}$ & No \\
\hline Composition & $\begin{array}{l}\text { Composition is built from simpler rules } \\
\text { using brackets, "(" and ")" for allocating } \\
\text { priorities to the operators: Enable " }>>\text { " } \\
\text { Disable "[>" Parallel " } \| \text { " and Choice } \\
\text { "[]". }\end{array}$ & $\begin{array}{l}\text { Allows composition } \\
\text { trying to reduce the } \\
\text { complexity of the } \\
\text { models } i^{*} \text {. }\end{array}$ & $\begin{array}{l}\text { Supports composition for } \\
\text { UML class, sequence and } \\
\text { state diagrams using graph } \\
\text { transformations (all } \\
\text { composition mechanisms are } \\
\text { from original MATA). }\end{array}$ & $\begin{array}{l}\text { Deals with scenario } \\
\text { variability as a composition } \\
\text { of different artifacts: SPL } \\
\text { UC \&, feature models, } \\
\text { product configuration, \& } \\
\text { configuration knowledge. }\end{array}$ \\
\hline
\end{tabular}


The set of comparison criteria is taken from [1]: Conflict resolution (conflicts are inevitable and can arise between the requirements, functional or non-functional); Heuristics (this is a set of steps aimed at facilitating access to new theoretical developments or discoveries, in our case to discover features); Tool support (the approach presents a support tool, for requirements management in support of its architecture, traceability, or its evolution); Modeling requirements (activities to capture the functional requirements, of a product line and their dependencies on each other); Modeling features (consist of activities to identify, study and describe the features relevant to a given domain); Modeling scenarios (include not only the functionality of systems and their interactions with users, but also aspects); Composition (analyzes the composition in the approach) and Feature interaction (occurs when the integration of two features would modify the behavior of one or both).

In summary, our approach has the following advantages: it provides a sound set of heuristics to derive a feature model that takes into account the identification of crosscutting concerns at domain and application engineering levels; a tool that offers a systematic and automatic way to identify common and variable features and a multi-criteria based method (AHP) to identify and resolve conflicts at a more abstract level.

\section{CONCLUSION AND FUTURE WORK}

PLAORA is an aspect-oriented approach that supports elicitation and analysis of requirements for SPL at domain and application engineering levels. It offers a set of heuristics to automatically derive feature model from aspect-oriented requirements descriptions. This is done automatically by the extension performed on the AORA tool. Aspect-orientation mechanisms were very useful in the definition of PLAORA to identify crosscutting features and consequently obtain a more modularized feature model. It brings to the community several advantages, as the comparison Table 3 shows.

As future work we need to work on the scalability of the model. We are planning to implement two different views of the system (functional and non-functional) to partially achieve this. Our final goal is to use lexical analysis and text mining to ultimately interpret the text offered by the AORA templates to extract initial the initial feature model. The resulting approach needs to be then applied to real case studies.

\section{ACKNOWLEDGMENTS}

This work was partially funded by the European AMPLE project and FCT MCTES.

\section{REFERENCES}

[1] AMPLE, “Ample Project”, http://www.ample-project.net/. Last access: August 2010.

[2] Baniassad, E., Clements, P., Araújo, J., Moreira, A., Rashid, A., Tekinerdogan, B. Discovering Early Aspects. IEEE Software, Vol 23(1), 2006.

[3] Bonifácio, R., Borba, P. Modeling Scenario Variability as Crosscutting Mechanisms. AOSD'09, USA, 2009.
[4] Brito, I., Vieira, F., A. Moreira, A., Ribeiro, R. Handling Conflicts in Aspectual Requirements Compositions, Transactions on AOSD, Vol 4620, 2007, pp. 144-166.

[5] Brito, I. Aspect-Oriented Requirements Analysis. PhD Thesis. Universidade Nova de Lisboa, Portugal, 2008.

[6] Chung, L., Nixon, B., Yu, E., Mylopoulos, J. NonFunctional Requirements in Software Engineering. Kluwer, 2000.

[7] Classen, A., Heymans, P., Laney, R., Nuseibeh, B., Tun, T. 1st International Workshop on Variability Modelling of Software-intensive Systems. Limerick. Ireland, 2001.

[8] Czarnecki, K., Helsen, S., Eisenecker, U. Staged Configuration Through Specialization and Multi-Level Configuration of Feature Models. SPLC'04 Conference. Boston, USA, 2004.

[9] Finkelstein, A., Sommerville, I. The Viewpoints FAQ. Software Engineering Journal: Special Issue on Viewpoints for Software Engineering. IEE/BCS, 1996.

[10] Gomaa, H. Designing Software Product Lines with UML: From Use Cases to Pattern based Software Architectures. Addison-Wesley, 2004.

[11] IEEE 1471: Recommended Practice for Architectural Description of Software-Intensive Systems. IEEE Computer Society, 2000.

[12] Jacobson, I., Chirsterson, M., Jonsson, P., Overgaard, G. Object-Oriented Software Engineering - a Use Case Driven Approach. Addison-Wesley, 1992.

[13] Jayaraman, P., Whittle, J., Elkhodary, AM., Gomaa, H. Model Composition in Product Lines and Feature Interaction Detection Using Critical Pair Analysis. MoDELS'07 Conference, Springer, 2007.

[14] Kang, K., Cohen, S., Hess, J., Nowak, W., Peterson, S. Feature-Oriented Domain Analysis (FODA) Feasibility Study. Tech. Rep., CMU/SEI-90-TR-021, USA, 1990.

[15] Kotonya, G., Sommerville, I. Requirements Engineering: Processes and Techniques. John Wiley, 1998.

[16] Pohl, K., Böckle, G., Van Der Linder F. Software Product Line Engineering Foundations, Principles, and Techniques. Springer, 2005.

[17] Rashid, A., Moreira, A., Araújo, J. Modularization and Composition of Aspectual Requirements. AOSD'03 Conference. Boston, EUA, 2003.

[18] Silva, C., Alencar, F., Araújo, J., Moreira, A., Castro, J.: Tailoring an Aspectual Goal-Oriented Approach to Model Features. SEKE'08 Conference. California EUA, 2008.

[19] Saaty, T. The Analytic Hierarchy Process. McGraw-Hill, 1980.

[20] Yu, Y., Leite, J., Lapouchnian, A., Mylopoulos, J. Configuring features with stakeholder goals. $A C M$ Symposium on Applied computing, 2008. 